\title{
Dyadic Torsion of Elliptic Curves
}

\author{
Jeffrey Yelton
}

May 3, 2018

\begin{abstract}
Let $k$ be a field of characteristic 0 , and let $\alpha_{1}, \alpha_{2}$, and $\alpha_{3}$ be algebraically independent and transcendental over $k$. Let $K$ be the transcendental extension of $k$ obtained by adjoining the elementary symmetric functions of the $\alpha_{i}$ 's. Let $E$ be the elliptic curve defined over $K$ which is given by the equation $y^{2}=\left(x-\alpha_{1}\right)\left(x-\alpha_{2}\right)\left(x-\alpha_{3}\right)$. We define a tower of field extensions $K=K_{0}^{\prime} \subset K_{1}^{\prime} \subset K_{2}^{\prime} \subset \ldots$ by giving recursive formulas for the generators of each $K_{n}^{\prime}$ over $K_{n-1}^{\prime}$. We show that $K_{\infty}^{\prime}$ is a certain central subextension of the field $K\left(E\left[2^{\infty}\right]\right):=\bigcup_{n=0}^{\infty} K\left(E\left[2^{n}\right]\right)$, and a generator of $K\left(E\left[2^{\infty}\right]\right)$ over $K_{\infty}^{\prime}\left(\mu_{2}\right)$ is given. Moreover, if we assume that $k$ contains all 2-power roots of unity, for each $n$, we show that $K\left(E\left[2^{n}\right]\right)$ contains $K_{n}^{\prime}$ and is contained in a certain quadratic extension of $K_{n+1}^{\prime}$.
\end{abstract}

\section{Introduction}

Let $k$ be a field of characteristic 0 which contains all 2-power roots of unity. Let $K$ be the transcendental extension of $k$ obtained by adjoining the coefficients of the cubic polynomial $\left(x-\alpha_{1}\right)\left(x-\alpha_{2}\right)\left(x-\alpha_{3}\right)$, where $\alpha_{1}, \alpha_{2}$, and $\alpha_{3}$ are independent and transcendental over $k$. Fix an algebraic closure $\bar{K}$ of $K$. Suppose that $E$ is the elliptic curve over $K$ given by the Weierstrass equation

$$
y^{2}=\left(x-\alpha_{1}\right)\left(x-\alpha_{2}\right)\left(x-\alpha_{3}\right) .
$$

For any integer $n \geq 0$, let $E\left[2^{n}\right]$ be the subgroup of $E(\bar{K})$ of $2^{n}$-torsion points, and let $K_{n}$ be the extension of $K$ over which they are defined. (Note that $K_{0}=K$ and $K_{1}=K\left(\alpha_{1}, \alpha_{2}, \alpha_{3}\right)$.) Further, denote by $E\left[2^{\infty}\right]$ the subgroup of all 2-power torsion points and denote by $K_{\infty}$ the minimal (algebraic) extension of $K$ over which they are defined.

In this paper,

$$
T_{2}(E):=\lim _{\leftarrow n} E\left[2^{n}\right]
$$

will denote the 2 -adic Tate module of $E$; it is a free $\mathbb{Z}_{2}$-module of rank 2 . Let

$$
V_{2}(E):=T_{2}(E) \otimes \mathbb{Q}_{2} .
$$

Then $V_{2}(E)$ is a 2-dimensional vector space over $\mathbb{Q}_{2}$ which contains the rank$2 \mathbb{Z}_{2}$-lattice $T_{2}(E)$. Let $\mathcal{L}$ be the set of all $\mathbb{Z}_{2}$-lattices $\Lambda \subset V_{2}(E)$ such that 
$\Lambda \supset T_{2}(E)$ but $\Lambda \not \supset \frac{1}{2} T_{2}(E)$. Set $\Lambda_{0}=T_{2}(E)$. There is an obvious bijection between $\mathcal{L}$ and the set of cyclic subgroups of $E\left[2^{\infty}\right]$, given by sending an element $\Lambda \in \mathcal{L}$ to $\Lambda / \Lambda_{0}$, which is canonically identified with a cyclic subgroup $N<E\left[2^{n}\right]$ for any $n$ such that $\Lambda \subset \frac{1}{2^{n}} \Lambda_{0}$. For each $n \geq 0$, we denote by $\mathcal{L}_{n}$ the subset of $\mathcal{L}$ consisting of lattices $\Lambda$ such that $\Lambda / \Lambda_{0}$ is cyclic of order $2^{n}$ (or equivalently, $\Lambda$ corresponds to a maximal cyclic subgroup of $E\left[2^{n}\right]$ under the above bijection), and let $\mathcal{L}_{\leq n}:=\bigcup_{0 \leq m \leq n} \mathcal{L}_{m}$ and $\mathcal{L}_{\geq n}:=\bigcup_{m \geq n} \mathcal{L}_{m}$

We now endow $\mathcal{L}$ with the structure of a graph by saying that two lattices in $\mathcal{L}$ are connected by an edge if one is contained in the other and the induced quotient of $\mathbb{Z}_{2}$-modules is isomorphic to $\mathbb{Z} / 2 \mathbb{Z}$. It is easy to show that this graph is isomorphic to a certain Bruhat-Tits tree described in 3, $\S 1.1$. In particular, $\mathcal{L}$ is a 3 -regular tree. We designate $\Lambda_{0}$ as the root, and observe that for each $n \geq 0, \mathcal{L}_{n}$ is the set of all vertices of distance $n$ from the root. The following properties immediately follow.

Proposition 1.1. a) Every vertex $\Lambda \in \mathcal{L}_{n}$ with $n \geq 1$ has a unique "parent" vertex; that is, a vertex $\tilde{\Lambda} \in \mathcal{L}_{n-1}$ which is connected to $\Lambda$ by an edge. Equivalently, $\tilde{\Lambda}$ is the unique lattice in $\mathcal{L}$ such that $\Lambda \supset \tilde{\Lambda}$ and $\Lambda / \tilde{\Lambda} \cong \mathbb{Z} / 2 \mathbb{Z}$.

b) Every vertex $\Lambda \in \mathcal{L}_{n}$ with $n \geq 2$ has a unique "twin" vertex; that is, a vertex $\Lambda^{\prime} \in \mathcal{L}_{n}$ such that $\Lambda \neq \Lambda^{\prime}$ but $\Lambda$ and $\Lambda^{\prime}$ have the same parent.

There are exactly 3 vertices in $\mathcal{L}_{1}$, corresponding to the 3 subgroups of order 2 of $E[2]$, which are generated by the 2 -torsion points $\left(\alpha_{1}, 0\right),\left(\alpha_{2}, 0\right)$, and $\left(\alpha_{3}, 0\right)$. For $i=1,2,3$, write $\Lambda\left(\alpha_{i}\right)$ for the vertex in $\mathcal{L}_{1}$ corresponding to the subgroup $\left\langle\left(\alpha_{i}, 0\right)\right\rangle<E[2]$. For any $\Lambda \in \mathcal{L}_{\geq 1}$, write $\tilde{\Lambda}$ for its parent vertex, and for any $\Lambda \in \mathcal{L}_{\geq 2}$, write $\Lambda^{\prime}$ for its twin vertex, as in Proposition 1.1. For $\Lambda=\Lambda\left(\alpha_{i}\right) \in \mathcal{L}_{1}$, let $\Lambda^{\prime}=\Lambda\left(\alpha_{i+1}\right)$, where the index $i$ is considered as an element of $\mathbb{Z} / 3 \mathbb{Z}$.

In order to state the main theorem, we need to define an infinite algebraic extension of $K$ which is obtained by adjoining generators corresponding to each vertex of $\mathcal{L}$. These generators have to be defined recursively, so that for any $n \geq$ 2 , the generator corresponding to a vertex in $\mathcal{L}_{n}$ is given by an algebraic function of the generators corresponding to certain vertices in $\mathcal{L}_{n-1}$. This motivates the following definition.

Definition 1.2. A decoration on the tree $\mathcal{L}$ is a function $\Phi: \mathcal{L}_{\geq 1} \rightarrow \bar{K}$ with the following three properties:

I) For any $\Lambda \in \mathcal{L}_{\geq 1}, \Phi(\Lambda) \neq \Phi\left(\Lambda^{\prime}\right)$.

II) For $i \in \mathbb{Z} / 3 \mathbb{Z}, \Phi\left(\Lambda\left(\alpha_{i}\right)\right)=\alpha_{i+1}-\alpha_{i+2}$.

III) For every $\Lambda \in \mathcal{L}_{2}, \Psi(\Lambda)$ is a root of the quadratic polynomial

$$
x^{2}-2\left(2 \Psi\left((\tilde{\Lambda})^{\prime}\right)+\Psi(\tilde{\Lambda})\right) x+\Psi\left((\tilde{\Lambda})^{\prime}\right)^{2} \in \bar{K}[x],
$$

and for every $\Lambda \in \mathcal{L}_{n}$ with $n \geq 3, \Psi(\Lambda)$ is a root of the quadratic polynomial

$$
x^{2}-2\left(\Psi\left((\tilde{\Lambda})^{\prime}\right)-2 \Psi(\tilde{\Lambda})\right) x+\Psi\left((\tilde{\Lambda})^{\prime}\right)^{2} \in \bar{K}[x] .
$$

Proposition 1.3. a) A decoration on $\mathcal{L}$ exists. 

let

b) Let $\Psi$ be a decoration on $\mathcal{L}$. For $n \geq 0$, let $K_{n}^{\prime}=K\left(\{\Psi(\Lambda)\}_{\Lambda \in \mathcal{L}_{\leq n}}\right)$ and

$$
K_{\infty}^{\prime}=\bigcup_{n \geq 0} K_{n}^{\prime}=K\left(\{\Psi(\Lambda)\}_{\Lambda \in \mathcal{L}_{\geq 1}}\right)
$$

Then the algebraic extensions $K_{n}^{\prime} / K$ and $K_{\infty}^{\prime} / K$ do not depend on the choice of $\Psi$.

Proof. For each $N \geq 1$, define $F_{N}$ to be the set of all functions $\Psi: \mathcal{L}_{\geq 1} \cap \mathcal{L}_{\leq N} \rightarrow$ $\bar{K}$ that satisfy I, II, and III for $n \leq N$. Clearly, each $F_{N}$ is finite, and for each $N<N^{\prime}$, there is a map from $F_{N^{\prime}}$ to $F_{N}$ by restriction, so it will suffice to show that each $F_{N}$ is nonempty. By definition, $F_{1}$ is nonempty, and one can explicitly show that $F_{2}$ is nonempty and that any function $\Psi \in F_{2}$ takes nonzero values in $\bar{K}$. Now we prove inductively that $F_{N}$ is nonempty for $N \geq 3$ by showing that for each $N \geq 2$ and function $\Psi_{N} \in F_{N}$, there is a function $\Psi_{N+1} \in F_{N+1}$ taking nonzero values which restricts to $\Psi_{N}$. This amounts to showing that for each $\Lambda \in$ $\mathcal{L}_{n}$ with $n \geq 2$, the polynomial $x^{2}-2\left(\Psi\left(\Lambda^{\prime}\right)-2 \Psi(\Lambda)\right) x+\Psi\left(\Lambda^{\prime}\right)^{2}$ has two distinct, nonzero roots in $\bar{K}$. It is clear from property II and a little computation that this is true for $\Lambda \in \mathcal{L}_{2}$. Now assume inductively that this claim holds for all $\Lambda \in \mathcal{L}_{n-1}$ for some $n \geq 3$. Let $\Lambda \in \mathcal{L}_{n}$. If 0 were a root of $x^{2}-2\left(\Psi\left(\Lambda^{\prime}\right)-2 \Psi(\Lambda)\right) x+\Psi\left(\Lambda^{\prime}\right)^{2}$, then the constant coefficient $\left(\Psi\left(\Lambda^{\prime}\right)\right)^{2}$ would be 0 . But $\Psi\left(\Lambda^{\prime}\right)$ is a root of the polynomial $x^{2}-2\left(\Psi\left(\tilde{\Lambda}^{\prime}\right)-2 \Psi(\tilde{\Lambda})\right) x+\Psi\left(\tilde{\Lambda}^{\prime}\right)^{2}$, which by the inductive assumption, has nonzero roots. Thus, the polynomial $x^{2}-2\left(\Psi\left(\Lambda^{\prime}\right)-2 \Psi(\Lambda)\right) x+\Psi\left(\Lambda^{\prime}\right)^{2}$ has nonzero roots. Now suppose that its roots are equal. Then its discriminant $4\left(\Psi\left(\Lambda^{\prime}\right)-2 \Psi(\Lambda)\right)^{2}-4 \Psi\left(\Lambda^{\prime}\right)^{2}=16 \Psi(\Lambda)\left(\Psi(\Lambda)-\Psi\left(\Lambda^{\prime}\right)\right)$ is 0 , implying that either $\Psi(\Lambda)=0$ or $\Psi(\Lambda)=\Psi\left(\Lambda^{\prime}\right)$. But $\Psi(\Lambda)$ and $\Psi\left(\Lambda^{\prime}\right)$ are the two roots of the polynomial $x^{2}-2\left(\Psi\left(\tilde{\Lambda}^{\prime}\right)-2 \Psi(\tilde{\Lambda})\right) x+\Psi\left(\tilde{\Lambda}^{\prime}\right)^{2}$, and by the inductive assumption, they are distinct and nonzero, so we have a contradiction, thus proving part (a).

Let $\Psi$ and $\Psi^{\prime}$ be two functions on $\mathcal{L}_{\geq 1}$ taking values in $\bar{K}$, and choose any $n \geq 0$. Then it is easy to show by induction on $n$ that if $\Psi$ and $\Psi^{\prime}$ both satisfy I, II, and III, then there is a permutation $\Sigma$ on $\mathcal{L}_{n}$ such that $\Psi^{\prime}(\Lambda)=\Psi\left(\Lambda^{\Sigma}\right)$ for each $\Lambda \in \mathcal{L}_{n}$. Therefore, $K\left(\{\Psi(\Lambda)\}_{\Lambda \in \mathcal{L}_{n}}\right)=K\left(\left\{\Psi^{\prime}(\Lambda)\right\}_{\Lambda \in \mathcal{L}_{n}}\right)$ for each $n$, which immediately implies part (b).

We write $\rho_{2}: \operatorname{Gal}(\bar{K} / K) \rightarrow \operatorname{GL}\left(T_{2}(E)\right)=\operatorname{Aut}_{\mathbb{Z}_{2}}\left(T_{2}(E)\right)$ for the continuous homomorphism induced by the natural Galois action on $T_{2}(E)$, and denote its image by $G$. Similarly, for any integer $n \geq 0$, we write $\bar{\rho}_{2}^{(n)}: \operatorname{Gal}\left(K_{n} / K\right) \rightarrow$ $\operatorname{GL}\left(E\left[2^{n}\right]\right)$ for the homomorphism induced by the natural Galois action on $E\left[2^{n}\right]$, and denote its image by $\bar{G}^{(n)}$. Let $G(n)$ denote the kernel of the natural surjection $G \rightarrow \bar{G}^{(n)}$; it is the image under $\rho_{2}$ of the normal subgroup $\operatorname{Gal}\left(\bar{K} / K_{n}\right) \triangleleft \operatorname{Gal}(\bar{K} / K)$. Note that $G(0)=G$.

It follows from Corollary 1.2(b) and Remark 4.2(a) of [5] that $G$ contains the subgroup $\mathrm{SL}\left(T_{2}(E)\right) \subset G L\left(T_{2}(E)\right)$ of automorphisms of determinant 1. (This also follows from applying Hilbert's Irreducibility Theorem to results such as Corollary 1 of Chapter 6 , $\S 3$ of [1].) We write $-1 \in \mathrm{SL}\left(T_{2}(E)\right) \subseteq G(1)$ for the scalar automorphism which acts on $T_{2}(E)$ as multiplication by -1 . 
We fix a compatible system $\left\{\zeta_{2^{n}}\right\}_{n \geq 0}$ of $2^{n}$-th roots of unity. For any extension field $L$ of $K$, let $L\left(\mu_{2}\right)=\bigcup_{n=1}^{\infty} L\left(\zeta_{2^{n}}\right)$.

We are now ready to state the main theorem.

Theorem 1.4. Let an elliptic curve $E / K$ be defined as above, with Weierstrass roots $\alpha_{1}, \alpha_{2}$, and $\alpha_{3}$, and define $K_{\infty}^{\prime}$ as in Proposition $1.3(b)$.

a) Choose $i, j \in\{1,2,3\}$ with $i \neq j$, and choose an element $\sqrt{\alpha_{i}-\alpha_{j}} \in \bar{K}$ whose square is $\alpha_{i}-\alpha_{j}$. Then we have

$$
K_{\infty}=K_{\infty}^{\prime}\left(\sqrt{\alpha_{i}-\alpha_{j}}\right)\left(\mu_{2}\right) .
$$

b) The element of $\operatorname{Gal}\left(\bar{K} / K_{1}\right)$ whose image under $\rho_{2}$ is $-1 \in G(1)$ acts on $K_{\infty}$ by fixing $K_{\infty}^{\prime}\left(\mu_{2}\right)$ and taking $\sqrt{\alpha_{i}-\alpha_{j}}$ to $-\sqrt{\alpha_{i}-\alpha_{j}}$ for $1 \leq i, j \leq 3, i \neq j$.

The next section is dedicated to a proof of Theorem[1.4. The idea behind the proof is to construct $K_{\infty}^{\prime}$ as a compositum of fields of definition of certain elliptic curves that have a 2-power isogeny to $E$. It will then be shown that $K_{\infty}^{\prime} \subset K_{\infty}$ is the subextension corresponding to the subgroup of scalar automorphisms in $G$, and generators of $K_{\infty}$ over $K_{\infty}^{\prime}$ will be found. In $\S 3$, we will use Theorem 1.4 to obtain additional results (Theorems 3.1 and 3.2).

\section{Proof of the main theorem}

We assume the notation of $\S 1$. In particular, we retain the convention that for $\alpha_{i} \in\left\{\alpha_{1}, \alpha_{2}, \alpha_{3}\right\}$, we will write $\alpha_{i+1}$ or $\alpha_{i+2}$ as though $i \in \mathbb{Z} / 3 \mathbb{Z}$. For each $\Lambda \in \mathcal{L}$, by the discussion in $\S 1$, there is a corresponding cyclic subgroup of $E\left[2^{\infty}\right]$, which we will denote by $N_{\Lambda}$. Furthermore, we will often use "<" and ">" to indicate inclusion of subgroups of $E\left[2^{\infty}\right]$.

We will assign to each $\Lambda \in \mathcal{L}_{n}$ an elliptic curve $E_{\Lambda}$ and a $2^{n}$-isogeny $\phi_{\Lambda}$ : $E \rightarrow E_{\Lambda}$ whose kernel is $N_{\Lambda}$, which we will later show (Proposition 2.4(b)) is defined over $K\left(N_{\Lambda}\right)$. We will do this using a well-known isogeny of degree 2 (4), Chapter III, Example 4.5) which is defined over the field of definition of its kernel.

Set $E_{\Lambda_{0}}:=E$, and let $\phi_{\Lambda_{0}}: E \rightarrow E_{\Lambda_{0}}$ be the identity isogeny.

For any $z \in \bar{K}$, denote by $E_{z}$ the elliptic curve with Weierstrass equation given by

$$
y^{2}=\left(x-\alpha_{1}-z\right)\left(x-\alpha_{2}-z\right)\left(x-\alpha_{3}-z\right) .
$$

Let $t_{z}$ the isomorphism $E \rightarrow E_{z}$ sending $(x, y) \mapsto(x+z, y)$. Define, for any $\beta, \gamma \in K_{1}$, the elliptic curves $E_{\beta, \gamma}$ and $E_{\beta, \gamma}^{\prime}$ given by the following Weierstrass equations:

$$
\begin{gathered}
E_{\beta, \gamma}: y^{2}=x(x-\beta)(x-\gamma), \\
E_{\beta, \gamma}^{\prime}: y^{2}=x^{3}-2(\beta+\gamma) x^{2}+(\beta-\gamma)^{2} x .
\end{gathered}
$$

Let $\phi_{\beta, \gamma}: E_{\beta, \gamma} \rightarrow E_{\beta, \gamma}^{\prime}$ be the isogeny of degree 2 given by

$$
\phi_{\beta, \gamma}:(x, y) \mapsto\left(x-(\beta+\gamma)+\frac{\beta \gamma}{x}, y\left(1-\frac{\beta \gamma}{x^{2}}\right)\right) .
$$


Note that the kernel of $\phi_{\beta, \gamma}$ has as its only nontrivial element the 2-torsion point $(0,0) \in E_{\beta, \gamma}$. Now define, for $i \in \mathbb{Z} / 3 \mathbb{Z}$,

$$
\phi_{\Lambda\left(\alpha_{i}\right)}: E \rightarrow E_{\alpha_{i+1}-\alpha_{i}, \alpha_{i+2}-\alpha_{i}}^{\prime}, \phi_{\Lambda\left(\alpha_{i}\right)}:=\phi_{\alpha_{i+1}-\alpha_{i}, \alpha_{i+2}-\alpha_{i}} \circ t_{-\alpha_{i}} .
$$

We assign $E_{\Lambda\left(\alpha_{i}\right)}:=E_{\alpha_{i+1}-\alpha_{i}, \alpha_{i+2}-\alpha_{i}}^{\prime}$. Note that for each $i, \phi_{\Lambda\left(\alpha_{i}\right)}$ is an isogeny whose kernel is the order-2 cyclic subgroup $N_{\Lambda\left(\alpha_{i}\right)}=\left\langle\left(\alpha_{i}, 0\right)\right\rangle$ of $E$.

From now on, for each $i \in \mathbb{Z} / 3 \mathbb{Z}$, let $a_{\Lambda\left(\alpha_{i}\right)}=\alpha_{i+1}-\alpha_{i+2}$. Now we may write the Weierstrass equation for $E_{\Lambda_{i}}$ as

$$
\begin{gathered}
y^{2}=x^{3}-2\left(\left(\alpha_{i+2}-\alpha_{i}\right)-\left(\alpha_{i}-\alpha_{i+1}\right)\right) x^{2}+\left(\alpha_{i+1}-\alpha_{i+2}\right)^{2} x \\
=x^{3}-2\left(2\left(\alpha_{i+2}-\alpha_{i}\right)+\left(\alpha_{i+1}-\alpha_{i+2}\right)\right) x^{2}+\left(\alpha_{i+1}-\alpha_{i+2}\right)^{2} x \\
=x^{3}-2\left(2 a_{\Lambda\left(\alpha_{i}\right)^{\prime}}+a_{\Lambda\left(\alpha_{i}\right)}\right) x^{2}+a_{\Lambda\left(\alpha_{i}\right)}^{2} x .
\end{gathered}
$$

Since 0 is a root of the cubic in the above equation, we know that $(0,0) \in$ $E_{\Lambda\left(\alpha_{i}\right)}[2]$, and it is easy to verify that in fact, $(0,0)$ is the image of both points in $E[2] \backslash N_{\Lambda\left(\alpha_{i}\right)}$. It follows that the inverse image of $\langle(0,0)\rangle<E_{N_{\Lambda\left(\alpha_{i}\right)}}[2]$ under $\phi_{N_{\Lambda\left(\alpha_{i}\right)}}$ is $E[2]$. Then the inverse images of the other two order-2 subgroups of $E_{N_{\Lambda\left(\alpha_{j}\right)}}(\bar{K})$ under $\phi_{N_{\Lambda\left(\alpha_{j}\right)}}$ are the two cyclic order-4 subgroups of $E(\bar{K})$ which contain $N_{\Lambda\left(\alpha_{j}\right)}$. It follows that these cyclic order-4 subgroups must be $N_{\Lambda}$ and $N_{\Lambda^{\prime}}$, where $\Lambda$ and $\Lambda^{\prime}$ are twin vertices in $\mathcal{L}_{2}$ whose parent vertex is $\Lambda\left(\alpha_{i}\right)$. Let $a_{\Lambda}$ (resp. $a_{\Lambda^{\prime}}$ ) be the (nonzero) root of the cubic in the above equation such that $\phi_{N_{\Lambda\left(\alpha_{i}\right)}}$ takes $N_{\Lambda}$ (resp. $\left.N_{\Lambda^{\prime}}\right)$ to the subgroup $\left\langle\left(a_{\Lambda}, 0\right)\right\rangle$ (resp. $\left\langle\left(a_{\Lambda^{\prime}}, 0\right)\right\rangle$ ) of $E_{N_{\Lambda\left(\alpha_{i}\right)}}(\bar{K})$. Now, using the notation of above, we have the elliptic curve $E_{-a_{\Lambda}, a_{\Lambda^{\prime}}-a_{\Lambda}}^{\prime}$ and the isogeny $\phi_{-a_{\Lambda}, a_{\Lambda^{\prime}}-a_{\Lambda}} \circ t_{-a_{\Lambda}}: E_{N_{\Lambda\left(\alpha_{i}\right)}} \rightarrow E_{-a_{\Lambda}, a_{\Lambda^{\prime}}-a_{\Lambda}}^{\prime}$. Its kernel is $\left\langle a_{\Lambda}, 0\right\rangle$. Therefore, if we assign $E_{N_{\Lambda}}:=E_{-a_{\Lambda}, a_{\Lambda^{\prime}}-a_{\Lambda}}^{\prime}$ and

$$
\phi_{N_{\Lambda}}:=\phi_{-a_{\Lambda}, a_{\Lambda^{\prime}}-a_{\Lambda}} \circ t_{-a_{\Lambda}} \circ \phi_{N_{\Lambda\left(\alpha_{j}\right)}}: E \rightarrow E_{N_{\Lambda}},
$$

then $\phi_{N_{\Lambda}}$ has kernel $N_{\Lambda}$. Its Weierstrass equation can be written as

$$
\begin{gathered}
y^{2}=x^{3}-2\left(\left(-a_{\Lambda}\right)+\left(a_{\Lambda^{\prime}}-a_{\Lambda}\right)\right) x^{2}+\left(\left(-a_{\Lambda}\right)-\left(a_{\Lambda^{\prime}}-a_{\Lambda}\right)\right)^{2} x \\
=x^{3}-2\left(a_{\Lambda^{\prime}}-2 a_{\Lambda}\right) x^{2}+a_{\Lambda^{\prime}}^{2} x .
\end{gathered}
$$

Thus, we have defined the desired $E_{N_{\Lambda}}$ and $\phi_{N_{\Lambda}}$ for all $v \in \mathcal{L}_{2}$.

We define the desired $a_{\Lambda}, \phi_{\Lambda}$, and $E_{\Lambda}$ for any $\Lambda \in \mathcal{L}_{\geq 3}$ in a similar manner, using induction. The idea is as follows. Assume that for some $n \geq 2$ we have defined $\phi_{\Lambda}, E_{\Lambda}$, and $a_{\Lambda}$ for all $\Lambda \in \mathcal{L}_{n}$ with the above properties, and choose a vertex $\lambda \in \mathcal{L}_{n+1}$ whose parent is $\Lambda$. Then since the Weierstrass equation of $E_{\Lambda}$ is given by (11), we may define $a_{\lambda}$ (resp. $a_{\lambda^{\prime}}$ ) to be the (nonzero) root of the cubic in (11) such that $\phi_{\Lambda}$ takes $N_{\lambda}$ (resp. $N_{\lambda^{\prime}}$ ) to the subgroup $\left\langle\left(a_{\lambda}, 0\right)\right\rangle$ (resp. $\left.\left\langle\left(a_{\lambda^{\prime}}, 0\right)\right\rangle\right)$ of $E_{\Lambda}(\bar{K})$. We make the assignments $E_{\lambda}:=E_{-a_{\lambda}, a_{\lambda^{\prime}}-a_{\lambda}}^{\prime}$ and

$$
\phi_{\lambda}:=\phi_{-a_{\lambda}, a_{\lambda^{\prime}}-a_{\lambda}} \circ t_{-a_{\lambda}} \circ \phi_{\Lambda}: E \rightarrow E_{\lambda},
$$

and check that $a_{\lambda}, \phi_{\lambda}$, and $E_{\lambda}$ have the desired properties (in particular, the kernel of $\phi_{\lambda}$ is $N_{\lambda}$ ). Since the parent of every vertex $\mathcal{L}_{n+1}$ is a vertex in $\mathcal{L}_{n}$, it 
follows that through the method described above, we have defined the desired $E_{\Lambda}$ and $\phi_{\Lambda}$ for all $\Lambda \in \mathcal{L}_{n+1}$. In this way, $a_{\Lambda}, E_{\Lambda}$, and $\phi_{\Lambda} \in \bar{K}$ are defined for all $\Lambda \in \mathcal{L}_{\geq 1}$. Furthermore, for all $\Lambda \in \mathcal{L}_{\geq 1}$, we define $K_{\Lambda}$ to be the extension of $K$ obtained by adjoining the coefficients of the Weierstrass equation of $E_{\Lambda}$ given above.

Lemma 2.1. Using the above notation, define $\Psi: \mathcal{L}_{\geq 1} \rightarrow \bar{K}$ by setting $\Psi(\Lambda)=$ $a_{\Lambda}$ for $\Lambda \in \mathcal{L}_{\geq 1}$. Then $\Psi$ is a decoration on $\mathcal{L}$.

Proof. By construction, $\Psi$ satisfies properties II and III in Definition 1.2 (see the cubics in (9) and (11)). Finally, as in the proof of Proposition 1.3 the roots of the above quadratics must be distinct, fulfilling property I.

Definition 2.2. For any integer $n \geq 0$, define the extension $K_{n}^{\prime}$ of $K$ to be the compositum of the fields $K_{N_{v}}$ for all $v \in|\mathcal{L}|_{\leq n} \backslash\left\{v_{0}\right\}$. Define the extension $K_{\infty}^{\prime}$ of $\bar{K}$ to be the infinite compositum

$$
K_{\infty}^{\prime}:=\bigcup_{n \geq 0} K_{n}^{\prime}
$$

In this way, we obtain a tower of field extensions

$$
K=K_{0}^{\prime} \subset K_{1}^{\prime} \subset K_{2}^{\prime} \subset \ldots \subset K_{n}^{\prime} \subset \ldots
$$

with $K_{\infty}^{\prime}=\bigcup_{n \geq 0} K_{n}^{\prime}$. The following lemma shows that this notation is consistent with the notation set in the statement of Proposition 1.3(b).

Lemma 2.3. For any $n \geq 1, K_{n}^{\prime}=K\left(\{\Psi(v)\}_{\Lambda \in \mathcal{L}_{\leq n} \backslash\left\{\Lambda_{0}\right\}}\right)$ for any decoration $\Psi$ on $\mathcal{L}$, and $K_{\infty}^{\prime}=K\left(\{\Psi(v)\}_{\Lambda \in \mathcal{L}_{\geq 1}}\right)$ for any decoration $\Psi$ on $\mathcal{L}$.

Proof. For each $\Lambda \in|\mathcal{L}|_{n}$ with $n \geq 1$, let $\left\{\Lambda_{0}, \Lambda_{1}, \ldots, \Lambda_{n}=\Lambda\right\}$ be the sequence of vertices in the path of length $n$ from $v_{0}$ to $v$. Let $\tilde{K}_{\Lambda}$ denote the compositum of the fields $K_{\Lambda}$ for all $\Lambda \in\left\{\Lambda_{0}, \Lambda_{1}, \ldots, \Lambda_{n}\right\}$. We claim that

$$
\tilde{K}_{\Lambda}=K\left(\alpha_{1}, \alpha_{2}, \alpha_{3},\left\{a_{\Lambda_{m}}\right\}_{1 \leq m \leq n}\right) .
$$

By Definition 2.2, Propositions 1.3(b), and Lemma 2.1, this suffices to prove the statement of the lemma.

The claim is trivial for $n=1$. Now assume inductively that the statement holds for some $n \geq 1$ and all $\Lambda \in \mathcal{L}_{n}$. Choose any $\Lambda \in \mathcal{L}_{n+1}$. We may apply the inductive assumption to $\tilde{\Lambda} \in \mathcal{L}_{n}$. We know that $E_{\Lambda}$ is given by a Weierstrass equation of the form (9) or (11) and is therefore defined over $K\left(a_{v}, a_{v^{\prime}}\right)$. But $a_{v} a_{v^{\prime}}$ is a coefficient of $E_{\tilde{\Lambda}}$, and so the only element that we need to adjoin to $\tilde{K}_{\tilde{\Lambda}}=K\left(\alpha_{1}, \alpha_{2}, \alpha_{3},\left\{a_{\Lambda_{m}}\right\}_{1 \leq m \leq n-1}\right)$ to obtain $\tilde{K}_{\Lambda}$ is $a_{\Lambda}$. Moreover, $a_{\Lambda}$ does lie in this extension, since $-\left(a_{\Lambda}+a_{\Lambda^{\prime}}\right)$ is a coefficient in the equation for $E_{\Lambda}$ and $2\left(2 a_{\Lambda^{\prime}}+a_{\Lambda}\right)\left(\right.$ resp. $\left.2\left(a_{\Lambda^{\prime}}-2 a_{\Lambda}\right)\right)$ is a coefficient of $E_{\tilde{\Lambda}}$ if $n=1$ (resp. $\left.n \geq 2\right)$. Thus, we have proved the claim for $n+1$. 
Proposition 2.4. With the above notation,

a) the isogeny $\phi_{N_{v}}$ is defined over $K\left(N_{v}\right)$, and $K_{N_{v}} \subseteq K\left(N_{v}\right)$,

b) for all $n \geq 0, K_{n}^{\prime} \subseteq K_{n}$, and equality holds for $n=0,1$.

Proof. First of all, for $i \in \mathbb{Z} / 3 \mathbb{Z}, E_{\Lambda\left(\alpha_{i}\right)}$ and $\phi_{\Lambda\left(\alpha_{i}\right)}$ are defined over $K\left(\alpha_{i+1}-\right.$ $\left.\alpha_{i}, \alpha_{i+2}-\alpha_{i}\right)=K\left(\alpha_{i}\right)$. This implies the equality in the $n=1$ case of the statament in part (b) (the equality in the $n=0$ case is trivial). It also proves part (a) for $\Lambda \in \mathcal{L}_{1}$, since $K\left(N_{\Lambda\left(\alpha_{i}\right)}\right)=K\left(\alpha_{i}\right)$ for each $i \in \mathbb{Z} / 3 \mathbb{Z}$.

Now assume inductively that for some $n \geq 1$ and all $\Lambda \in \mathcal{L}_{n}, \phi_{\Lambda}$ is defined over $K\left(N_{\Lambda}\right)$ and $K_{N_{\Lambda}} \subseteq K\left(N_{\Lambda}\right)$. Choose any $\Lambda \in \mathcal{L}_{n+1}$. We may apply the inductive assumption to $\tilde{\Lambda}$, since $\tilde{\Lambda} \in \mathcal{L}_{n}$. Let $P$ be a generator of the cyclic order- $2^{n+1}$ subgroup $N_{\Lambda}$. Then $P$ has coordinates in $K\left(N_{\Lambda}\right)$ and $\phi_{\tilde{\Lambda}}$ is defined over $K\left(N_{\tilde{\Lambda}}\right) \subseteq K\left(N_{\Lambda}\right)$, and it follows that $\phi_{\tilde{\Lambda}}(P)=\left(a_{\Lambda}, 0\right)$ has coordinates in $K\left(N_{\Lambda}\right)$. Thus, $a_{\Lambda} \in K\left(N_{\Lambda}\right)$. But $a_{\Lambda} a_{\Lambda^{\prime}}$ is a coefficient of $E_{\tilde{\Lambda}}$ and $K_{\tilde{\Lambda}} \subseteq K\left(N_{\tilde{\Lambda}}\right)$, so $a_{\Lambda^{\prime}} \in K\left(N_{\Lambda}\right)$ also. By construction, $\phi_{-a_{\Lambda}, a_{\Lambda^{\prime}}-a_{\Lambda}} \circ t_{-a_{\Lambda}}$ is defined over $K\left(a_{\Lambda}, a_{\Lambda^{\prime}}\right)$, so $\phi_{\Lambda}=\phi_{-a_{\Lambda}, a_{\Lambda^{\prime}}-a_{\Lambda}} \circ t_{-a_{\Lambda}} \circ \phi_{\tilde{\Lambda}}$ is defined over $K\left(N_{\tilde{\Lambda}}\right)\left(a_{\Lambda}, a_{\Lambda^{\prime}}\right) \subseteq K\left(N_{\Lambda}\right)$. Moreover, the Weierstrass equation (11) of $E_{\Lambda}=$ $E_{-a_{\Lambda}, a_{\Lambda^{\prime}}-a_{\Lambda}}^{\prime}$ has coefficients in $K\left(a_{\Lambda}, a_{\Lambda^{\prime}}\right) \subseteq K\left(N_{\Lambda}\right)$, and so $K_{\Lambda} \subseteq K\left(N_{\Lambda}\right)$, thus proving part (a).

Now part (a) and the fact that $K_{n}^{\prime}$ is the compositum of the fields $K_{\Lambda}$ for all $\Lambda \in \mathcal{L}_{\leq n} \backslash\left\{\Lambda_{0}\right\}$ imply that $K_{n}^{\prime}$ is contained in the compositum of the extensions $K\left(N_{\Lambda}\right)$ for all $\Lambda \in \mathcal{L}_{\leq n} \backslash\left\{\Lambda_{0}\right\}$. Since $\left\{N_{\Lambda}\right\}_{\Lambda \in \mathcal{L}_{\leq n}}$ is the set of all cyclic subgroups of $E\left[2^{n}\right]$ and therefore generates $E\left[2^{n}\right]$, this compositum is $K_{n}$. Thus, $K_{n}^{\prime} \subseteq K_{n}$, which is the statement of (b).

Next we want to determine how the absolute Galois group of $K$ acts on the $a_{\Lambda}$ 's defined above. In order to describe this Galois action, we will adopt the following notation. The automorphism group $\mathrm{GL}\left(T_{2}(E)\right)$ acts on the set of rank-2 $\mathbb{Z}_{2}$-lattices in $V_{2}(E)$ by left multiplication, and this action stabilizes $\mathcal{L}$, since $\operatorname{GL}\left(T_{2}(E)\right.$ ) fixes $T_{2}(E)$. (In fact, this action of $\operatorname{GL}\left(T_{2}(E)\right)$ on $\mathcal{L}$ is the action of $\operatorname{Aut}_{\mathbb{Z}_{2}}\left(T_{2}(E)\right) \subset \operatorname{Aut}_{\mathbb{Q}_{2}}\left(V_{2}(E)\right)$ on the Bruhat-Tits tree as described in [3, §1.2.) In particular, each $\mathcal{L}_{n}$ is invariant under the action. Recall the definitions of $G$ and $\bar{G}^{(n)}$ from $\S 1$. We will denote the action of $G \subset \operatorname{GL}\left(T_{2}(E)\right)$ on $\Lambda$ by $(s, \Lambda) \mapsto s \cdot \Lambda$ for an automorphism $s$ and vertex $\Lambda$. Note that this action of $G$, when restricted to $\mathcal{L}_{\leq n}$, factors through $G \rightarrow \bar{G}^{(n)}$. We similarly denote the resulting action of $\bar{G}^{(n)}$ on the subtree $\Lambda_{n}$ by $(\bar{s}, \Lambda) \mapsto \bar{s} \cdot \Lambda$ for an automorphism $\bar{s}$ and a vertex $\Lambda$.

Recall that $\rho_{2}: \operatorname{Gal}(\bar{K} / K) \rightarrow \operatorname{GL}\left(T_{2}(E)\right)\left(\right.$ resp. $\bar{\rho}_{2}^{(n)}: \operatorname{Gal}\left(K_{n} / K\right) \rightarrow$ $\left.\operatorname{GL}\left(E\left[2^{n}\right]\right)\right)$ is the homomorphism induced by the Galois action on $T_{2}(E)$ (resp. the Galois action on $E\left[2^{n}\right]$, for each $\left.n \geq 0\right)$. For any Galois element $\sigma \in$ $\operatorname{Gal}(\bar{K} / K)$ and vertex $\Lambda$ of $\mathcal{L}$, let $\Lambda^{\sigma}:=\rho_{2}(\sigma) \cdot \Lambda$. If $\Lambda \in \mathcal{L}_{\leq n}$ for some $n \geq 1$, then let $\Lambda^{\sigma \mid K_{n}}:=\bar{\rho}_{2}^{(n)}(\sigma) \cdot \Lambda$.

Lemma 2.5. For any $\sigma \in \operatorname{Gal}\left(\bar{K} / K_{1}\right)$ and vertex $\Lambda$, we have $a_{v}^{\sigma}=a_{v^{\sigma}}$. If $\Lambda \in \mathcal{L}_{\leq n}$, then $a_{\Lambda}^{\left.\sigma\right|_{K_{n}}}=a_{\Lambda^{\sigma \mid K_{n}}}$. 
Proof. Choose any $\sigma \in \operatorname{Gal}\left(\bar{K} / K_{1}\right)$. We will prove that $a_{\Lambda}^{\sigma}=a_{\Lambda^{\sigma}}$ for all $\Lambda \in \mathcal{L}_{n}$ for each $n \geq 1$. The claim is trivially true for $n=1$. Moreover, in the $n=1$ case, $E_{\Lambda}$ and $\phi_{\Lambda}$ are clearly defined over $K_{1}$ and are therefore fixed by $\sigma$. In particular, for any $\Lambda \in \mathcal{L}_{1}, a_{\Lambda}^{\sigma}=a_{\Lambda^{\sigma}}, E_{\Lambda}^{\sigma}=E_{\Lambda^{\sigma}}$, and $\phi_{\Lambda}^{\sigma}=\phi_{\Lambda^{\sigma}}$. Now choose $\Lambda \in \mathcal{L}_{2}$. Then $a_{\Lambda}$ is a nonzero root of (9), which is the Weierstrass cubic for $E_{\tilde{\Lambda}}$. Let $P$ be a generator of the cyclic order-4 subgroup $N_{\Lambda}$; then $\phi_{\tilde{\Lambda}}(P)=\left(a_{\Lambda}, 0\right) \in E_{\tilde{\Lambda}}[2]$, by the above construction of $a_{\Lambda}$. So we have

$$
\phi_{N_{\tilde{v} \sigma}}\left(P^{\sigma}\right)=\phi_{N_{\tilde{v}}}^{\sigma}\left(P^{\sigma}\right)=\left(\phi_{N_{\tilde{v}}}(P)\right)^{\sigma}=\left(\alpha_{v}^{\sigma}, 0\right) .
$$

But $P^{\sigma}$ generates $N_{v}^{\sigma}=N_{v^{\sigma}}$. By the above construction, we have $\phi_{N_{\tilde{v}^{\sigma}}}\left(P^{\sigma}\right)=$ $\left(a_{v^{\sigma}}, 0\right)$. Then (13) implies that $a_{v}^{\sigma}=a_{v^{\sigma}}$.

Now assume inductively that for some $n \geq 2$ and $\sigma \in \mathcal{L}_{n}, a_{\Lambda}^{\sigma}=a_{\Lambda^{\sigma}}$, $E_{\Lambda}^{\sigma}=E_{v^{\sigma}}$, and $\phi_{\Lambda}^{\sigma}=\phi_{\Lambda^{\sigma}}$, and choose any $\Lambda \in \mathcal{L}_{n+1}$. Then $a_{\Lambda}$ is a nonzero root of (11), and by a similar argument, again $a_{\Lambda}^{\sigma}=a_{\Lambda^{\sigma}}$. This inductive argument proves the first statement, and the second statement follows immediately.

Proposition 2.6. For all $n \geq 1$, the image of $\operatorname{Gal}\left(K_{n} / K_{n}^{\prime}\right)\left(\right.$ resp. $\operatorname{Gal}\left(K_{\infty} / K_{\infty}^{\prime}\right)$ ) under $\bar{\rho}_{2}^{(n)}$ (resp. $\left.\rho_{2}\right)$ coincides with the subgroup of scalar automorphisms in $G$ (resp. in $\left.\bar{G}^{(n)}\right)$.

Remark 2.7. Since each $\sigma \in \operatorname{Gal}(\bar{K} / K)$ takes $\zeta_{8}$ to $\zeta_{8}^{\operatorname{det}\left(\bar{\rho}_{2}^{(3)}(\sigma)\right)}$, all scalar automorphisms in $G$ must fix $\zeta_{8}$. Thus, Proposition 2.6 implies that $K_{\infty}$ contains the 8 th roots of unity.

Proof. Fix $n \geq 1$. Since $K_{n}^{\prime} \supseteq K_{1}$ for each $n \geq 1$, we only need to consider the Galois subgroup $\operatorname{Gal}\left(K_{n}^{\prime} / K_{1}\right) \subseteq \operatorname{Gal}\left(K_{n} / K_{1}\right)$. Proposition 2.3, with the help of Lemma 2.1] implies that $K_{n}^{\prime}$ is generated over $K_{1}$ by the elements $a_{\Lambda}$ for all $\Lambda \in \mathcal{L}_{\leq n} \backslash\left\{\Lambda_{0}\right\}$. Therefore, the elements of $\operatorname{Gal}\left(K_{\infty} / K_{1}\right)$ which fix $K_{n}^{\prime}$ are exactly those which fix all of the elements $a_{\Lambda}$ for $\Lambda \in \mathcal{L}_{\leq n} \backslash\left\{\Lambda_{0}\right\}$. By Lemma 2.5. these are the Galois elements which fix every vertex in $\mathcal{L}_{\leq n}$. It is easy to see that an element of $\mathrm{GL}\left(E\left[2^{n}\right]\right)$ fixes every vertex in $\mathcal{L}_{n}$ if and only if it is a scalar automorphism. Thus, the image of $\operatorname{Gal}\left(K_{n} / K_{n}^{\prime}\right)$ coincides with the subgroup of scalars, as desired. A similar argument proves the analogous statement for $\operatorname{Gal}\left(K_{\infty} / K_{\infty}^{\prime}\right)$.

From now on, for ease of notation, we set $a_{i}:=a_{\Lambda\left(\alpha_{i}\right)}=\alpha_{i+1}-\alpha_{i+2}$ for $i \in \mathbb{Z} / 3 \mathbb{Z}$. For each $a_{i}$, choose an element $\sqrt{a_{i}} \in \bar{K}$ whose square is $a_{i}$. Also, for $r \in \mathbb{Z}_{2}$, we will write $r \in \mathrm{SL}\left(T_{2}(E)\right.$ ) (resp. $r \in \mathrm{SL}\left(E\left[2^{n}\right]\right.$ ) for some $n$ ) for the scalar matrix corresponding to $r$ (resp. $r$ modulo $2^{n}$ ).

Note that, due to the Galois equivariance of the Weil pairing, the image of $\operatorname{Gal}\left(\bar{K} / K\left(\mu_{2}\right)\right)$ under $\rho_{2}$ coincides with $\mathrm{SL}\left(T_{2}(E)\right)$, and $K_{\infty} \supset K\left(\mu_{2}\right)$. The following proposition, together with Proposition 2.3. gives the statement of Theorem 1.4. 
Proposition 2.8. The extension $K_{\infty}^{\prime}\left(\mu_{2}\right) \supset K\left(\mu_{2}\right)$ corresponds to the subgroup $\{ \pm 1\} \triangleleft \mathrm{SL}\left(T_{2}(E)\right) \cong \operatorname{Gal}\left(K_{\infty} / K\left(\mu_{2}\right)\right)$. In fact,

$$
K_{\infty}=K_{\infty}^{\prime}\left(\sqrt{a_{i}}\right)\left(\mu_{2}\right)
$$

for any $i \in\{1,2,3\}$, and the Galois element corresponding to $-1 \in \mathrm{SL}\left(T_{2}(E)\right)$ acts by taking $\sqrt{a_{i}}$ to $-\sqrt{a_{i}}$.

Proof. If we replace $K$ with $K\left(\mu_{2}\right)$, it will suffice to assume that $K$ contains all 2-power roots of unity and to prove that $K_{\infty}=K_{\infty}^{\prime}\left(\sqrt{a_{i}}\right)$ for any $i \in\{1,2,3\}$ and that the Galois element corresponding to $-1 \in \mathrm{SL}\left(T_{2}(E)\right)$ acts as claimed.

Since $T_{2}(E)$ has rank 2, the determinant of any scalar $r \in \operatorname{Aut}_{\mathbb{Z}_{2}}\left(T_{2}(E)\right)$ is $r^{2} \in \mathbb{Z}_{2}$. Therefore, the only scalar automorphisms in $\mathrm{SL}\left(T_{2}(E)\right)$ are \pm 1 . Proposition 2.6 then implies that the image under $\rho_{2}$ of $\operatorname{Gal}\left(K_{\infty} / K_{\infty}^{\prime}\right)$ coincides with $\{ \pm 1\} \triangleleft \mathrm{SL}\left(T_{2}(E)\right)$.

It immediately follows that $K_{\infty}$ is generated over $K_{\infty}^{\prime}$ by any element of $K_{\infty}$ which is not fixed by the Galois automorphism $\sigma$ such that $\rho_{2}(\sigma)=-1 \epsilon$ $\operatorname{SL}\left(T_{2}(E)\right)$. Clearly, $\bar{\rho}_{2}^{(2)}\left(\left.\sigma\right|_{K_{2}}\right)=-1 \in \Gamma(2) / \Gamma(4)$. But setting $n=2$ in the statement of Proposition 2.6 implies that

$$
\operatorname{Gal}\left(K_{2} / K_{2}^{\prime}\right) \cong\{ \pm 1\}<\Gamma(2) / \Gamma(4),
$$

so any element in $K_{2} \backslash K_{2}^{\prime}$ will not be fixed by -1 . One checks using the formulas for $a_{\Lambda}$ with $v \in|\mathcal{L}|_{2}$ and using Proposition 2.3 for $n=2$ that

$$
K_{2}^{\prime}=K_{1}\left(\sqrt{a_{1} a_{2}}, \sqrt{a_{2} a_{3}}, \sqrt{a_{3} a_{1}}\right) .
$$

However, it can be verified through direct calculation that

$$
K_{2}=K_{1}\left(\sqrt{\alpha_{1}}, \sqrt{\alpha_{2}}, \sqrt{\alpha_{3}}\right) .
$$

Therefore, for each $i, \sqrt{a_{i}} \in K_{2} \backslash K_{2}^{\prime}$, so $\sqrt{a_{i}}$ can be used to generate $K_{\infty}$ over $K_{\infty}^{\prime}$. Moreover, since $a_{i} \in K_{1} \subset K_{\infty}^{\prime}$, it follows that -1 sends $\sqrt{a_{i}}$ to $-\sqrt{a_{i}}$.

\section{Some applications}

Theorem 3.1. Write $K\left(x\left(E\left[2^{n}\right]\right)\right)$ (resp. $K\left(x\left(E\left[2^{\infty}\right]\right)\right)$ ) for the extension of $K$ obtained by adjoining the $x$-coordinates of all elements of $E\left[2^{n}\right]$ (resp. $E\left[2^{\infty}\right]$ ). Then

a) $K_{n}=K\left(x\left(E\left[2^{n}\right]\right)\right)\left(\sqrt{a_{i}}\right)$ for all $n \geq 2$ and $i=1,2,3$;

b) $K_{n}^{\prime}\left(\zeta_{2^{n}}\right) \subseteq K\left(x\left(E\left[2^{n}\right]\right)\right)$ for all $n \geq 1$; and

c) $K_{\infty}^{\prime}\left(\mu_{2}\right)=K\left(x\left(E\left[2^{\infty}\right]\right)\right)$.

Proof. The Galois equivariance of the Weil pairing implies that $K_{n} \supset K\left(\zeta_{2^{n}}\right)$ for each $n \geq 0$ and that $K_{\infty} \supset K\left(\mu_{2}\right)$. Choose a basis of $T_{2}(E)$, so that we may identify $\operatorname{Gal}\left(K_{\infty} / K\right)$ with $\mathrm{GL}_{2}\left(\mathbb{Z}_{2}\right)$. For any $n \geq 1$, the subgroup of 
$\operatorname{Gal}\left(K_{n} / K\right)$ which fixes the $x$-coordinates of the points in $E\left[2^{n}\right]$ coincides with the subgroup whose image under $\bar{\rho}_{2}^{(n)}$ is identified with the matrices in $\mathrm{GL}_{2}\left(\mathbb{Z}_{2}\right)$ which send each point $P \in E\left[2^{n}\right]$ either to $P$ or to $-P$. The only such matrices are the scalar matrices \pm 1 . Thus, $K\left(x\left(E\left[2^{n}\right]\right)\right)$ is the subextension of $K_{n}$ fixed by $\{ \pm 1\} \triangleleft \mathrm{GL}_{2}\left(\mathbb{Z} / 2^{n} \mathbb{Z}\right)$, and similarly, $K\left(x\left(E\left[2^{\infty}\right]\right)\right)$ is the subextension of $K_{\infty}$ fixed by $\{ \pm 1\} \triangleleft \mathrm{GL}\left(T_{2}(E)\right)$. As in the proof of Proposition [2.8, $\sqrt{a_{i}}$ lies in $K_{2} \subseteq K_{n}$ and is not fixed by -1 for each $i$, hence the statement of part (a). Parts (b) and (c) then immediately follow from Proposition 2.6.

The description of $K_{\infty}^{\prime}$ given in the statement of Theorem 1.4 provides us with recursive formulas for the generators of $K_{n}^{\prime}$ for each $n \geq 0$. We will not similarly obtain formulas for the generators of each extension $K_{n}$, but the above results do give us a way of "bounding" each $K_{n}$, as follows:

Theorem 3.2. For each $n \geq 2$ and $i=1,2,3$,

$$
K_{n}^{\prime}\left(\sqrt{a_{i}}, \zeta_{2^{n}}\right) \subseteq K_{n} \subsetneq K_{n+1}^{\prime}\left(\sqrt{a_{i}}, \zeta_{2^{n+1}}\right),
$$

where the first inclusion is an equality if and only if $n=2$. Furthermore, $\left[K_{n}: K_{n}^{\prime}\left(\sqrt{a_{1}}, \zeta_{2^{n}}\right)\right]=2$ for $n \geq 3$, and $\left[K_{n+1}^{\prime}\left(\sqrt{a_{1}}, \zeta_{2^{n+1}}\right): K_{n}\right]=4$ for $n \geq 2$.

Proof. Fix $n \geq 2$ and $i \in\{1,2,3\}$. Proposition 2.4(b) and the inclusion $K_{n} \supset$ $K\left(\zeta_{2^{n}}\right)$ imply that $K_{n}^{\prime} \subset K_{n}$, and, as in the proof of Proposition 2.8, $\sqrt{a_{i}} \in$ $K_{2} \subseteq K_{n}$, thus implying the first inclusion. For $n=2$, it has already been shown in the proof of Proposition 2.8 that the inclusion is an equality. Since $\sqrt{a_{i}} \notin K_{\infty}^{\prime}\left(\mu_{2}\right)$, it follows that $\sqrt{a_{i}} \notin K\left(2^{i}\right)^{\prime}\left(\zeta_{2^{i}}\right)$ for any positive integer $i$. Note that by Proposition 2.6. for $n \geq 3, \operatorname{Gal}\left(K_{n} / K_{n}^{\prime}\left(\zeta_{2^{n}}\right)\right)$ is identified with the subgroup $\left\{ \pm 1, \pm\left(2^{n-1}+1\right)\right\} \triangleleft \mathrm{SL}_{2}\left(\mathbb{Z} / 2^{n} \mathbb{Z}\right)$, which is of order 4 . It follows that the degree of the first inclusion is 2 in this case. Equivalently, for all $n \geq 2$, $K_{n+1} \supset K_{n+1}^{\prime}\left(\sqrt{a_{1}}, \zeta_{2^{n+1}}\right)$ is an extension of degree 2 . We have $\left(\right.$ via $\left.\bar{\rho}_{2}^{(n+1)}\right)$ the following identifications:

$$
\begin{gathered}
\operatorname{Gal}\left(K_{n+1} / K\right) \cong G(2) / G\left(2^{n+1}\right), \\
\operatorname{Gal}\left(K_{n+1} / K_{n+1}^{\prime}\left(\zeta_{2^{n+1}}\right) \cong\left\langle-1,2^{n}+1\right\rangle \triangleleft G(2) / G\left(2^{n+1}\right) .\right.
\end{gathered}
$$

These imply that $\operatorname{Gal}\left(K_{n+1} / K_{n+1}^{\prime}\left(\sqrt{a_{i}}, \zeta_{2^{n+1}}\right)\right)$ is a subgroup of $\left\langle-1,2^{n}+1\right\rangle$ of order 2. Since $2^{n}+1$ fixes all of $K(4)$, which includes the element $\sqrt{a_{i}}$, we have $\operatorname{Gal}\left(K_{n+1} / K_{n+1}^{\prime}\left(\sqrt{\alpha_{i}}, \zeta_{2^{n+1}}\right)\right) \cong\left\langle 2^{n}+1\right\rangle \triangleleft \Gamma(2) / \Gamma\left(2^{n+1}\right)$. But this subgroup also leaves $K_{n}$ fixed, whence the second inclusion $K_{n} \subset K_{n+1}^{\prime}\left(\sqrt{a_{i}}, \zeta_{2^{n+1}}\right)$. The fact that $\left[K_{n+1}^{\prime}\left(\sqrt{a_{i}}\right): K_{n}\right]=4$ follows quickly from the fact that $\operatorname{Gal}\left(K_{n+1} / K_{n}\right) \cong$ $\left(G(n) \cap \mathrm{SL}\left(T_{2}(E)\right) / G\left(2^{n+1}\right)\right.$ is isomorphic to $(\mathbb{Z} / 2 \mathbb{Z})^{3}$ (see the proof of Corollary 2.2 of [2]) and therefore has order 8. 


\section{Acknowledgements}

The author would like to thank Yuri Zarhin and Mihran Papikian for their patience, and for insightful discussions which were extremely helpful in producing this work.

\section{References}

[1] Serge Lang. Elliptic functions. Springer, 1987.

[2] Masatoshi Sato. The abelianization of the level $d$ mapping class group. Journal of Topology, 3(4):847-882, 2010.

[3] Jean-Pierre Serre. Trees. Translated from the French original by John Stillwell. Corrected 2nd printing of the 1980 English translation. Springer Monographs in Mathematics. Springer-Verlag, Berlin, 2003.

[4] Joseph H Silverman. The arithmetic of elliptic curves, volume 106. Springer, 2009.

[5] Jeffrey Yelton. Images of 2-adic representations associated to hyperelliptic Jacobians. arXiv preprint arXiv:1410.2668, 2014. 